\title{
Refractive Outcomes for Combined Phacoemulsification and Glaucoma Drainage Procedure
}

\author{
Rajvi Mehta $\cdot$ Shizuka Tomatzu $\cdot$ Dingcai Cao $\cdot$ Alexander Pleet · \\ Alexander Mokhur · Ahmad A. Aref · Thasarat Sutabutr Vajaranant
}

Received: October 17, 2021 / Accepted: November 17, 2021 / Published online: December 4, 2021

(C) The Author(s) 2021

\section{ABSTRACT}

Introduction: To evaluate the refractive outcome of combined cataract extraction and glaucoma drainage device (GDD) surgery.

Methods: Patients who had undergone combined phacoemulsification with GDD surgery [Baerveldt, Abbott Medical, Abbott Park (IL) or Ahmed valve, New World Medical, Rancho Cucamonga (CA)] between June 2009 and August 2017 were included in the study. The main outcome measure evaluated was whether or not spherical equivalent (SE) between $\pm 1 \mathrm{D}$ from target refraction was achieved at 3-6 months postoperatively.

Results: The final analysis included 42 eyes of 38 patients who underwent combined phacoemulsification and GDD surgery. A refractive outcome of spherical equivalent (SE) between $\pm 1 \mathrm{D}$ of the target refraction was achieved in 30 of 42 eyes $(71.43 \%)$ at

R. Mehta · A. Pleet · A. A. Aref · T. S. Vajaranant ( $₫)$ Illinois Eye and Ear Infirmary, 1855 W. Taylor Street, M/C 648, Chicago, IL 60612, USA

e-mail: thasarat@uic.edu

S. Tomatzu - D. Cao $\cdot$ A. Mokhur

Department of Ophthalmology and Visual Sciences, University of Illinois College of Medicine, Chicago, IL, USA
3-6 months after surgery. Mean preoperative axial length $(\mathrm{AL})$ of eyes with postoperative $\mathrm{SE}$ outside $\pm 1 \mathrm{D}$ from target $(\mathrm{SD}=0.98, p=0.003$ ) was noted to be $25.37 \pm 0.98 \mathrm{~mm}$ (longer mean $\mathrm{AL})$ and that of eyes with $\mathrm{SE}$ between $\pm 1 \mathrm{D}(\mathrm{SD}=0.89, p=0.000)$ was found to be $23.34 \pm 0.89 \mathrm{~mm}$ (average mean $\mathrm{AL})$. Twelve (29\%) eyes were noted to have a mean $0.52 \mathrm{D}$ ( $\mathrm{SD}=0.49$; range $0.02-1.49$ ) of corneal astigmatism induced by combined surgery. Age, central corneal thickness, preoperative anterior chamber depth, and pre- and postoperative intraocular pressure did not significantly affect refractive outcomes.

Conclusion: Refractive outcomes within 1.00D of the target refraction were achieved in most patients undergoing a combined surgical approach. Longer AL was a risk factor among patients with refractive change $>1.00 \mathrm{D}$ from target.

Keywords: Cataract; Combined surgery; Glaucoma implant; Refractive outcome 


\section{Key Summary Points}

Refractive rehabilitation in patients undergoing combined cataract and glaucoma surgery depends on multiple pre- and postoperative factors.

This is the largest study which aimed to evaluate refractive outcomes after combined cataract extraction with glaucoma drainage device implantation.

Favorable refractive outcomes within 1.00D of the target refraction were noted in most patients.

Longer preoperative axial length (AL) was noted to be a risk factor in patients with refractive outcomes $>1.00 \mathrm{D}$ from target refraction, and this knowledge can help guide patient education, intraocular lens (IOL) selection, and the consent process in the preoperative period.

\section{INTRODUCTION}

Combined glaucoma and cataract surgery is frequently considered for patients with both clinically progressive glaucoma and visually significant cataract. Successful pseudophakic rehabilitation after combined glaucoma filtering surgery and cataract surgery requires accurate intraocular lens (IOL) power calculation, which depends on several factors including axial length $(\mathrm{AL})$, corneal curvature, and anterior chamber depth (ACD) [1, 3-7]. Studies have shown that postoperative anterior chamber depth and preoperative $\mathrm{AL}$ measurements are the largest contributors to refractive error (35\% and $17 \%$, respectively) after combined glaucoma and cataract surgery and that with-therule astigmatism is often induced after these combined procedures [1-9].

Law et al. published on the refractive outcomes in combined trabeculectomy with cataract extraction and reported no significant difference between the expected and observed refractive errors [10]. Tzu et al. were the first to report refractive outcomes in combined cataract extraction and glaucoma drainage device surgeries. Their study evaluated refractive outcomes in 43 total eyes which had undergone cataract extraction with either glaucoma drainage device (GDD) implantation or trabeculectomy, and they reported acceptable refractive outcomes (spherical equivalent between $-1.00 \mathrm{D}$ and $+0.50 \mathrm{D})$ in the majority their study cohort. Of these, 21 eyes (48\%) had undergone combined cataract extraction with GDD implantation. The authors recognized that a larger sample size would allow for a better understanding of the factors influencing these refractive results [11].

Given limited reports on this topic, our study was the largest study to date, designed to evaluate the refractive outcomes of combined cataract extraction and glaucoma drainage device surgery and further identify factors that were associated with refractive outcomes.

\section{METHODS}

\section{Study Population}

The University of Illinois (UIC) Institutional Review Board reviewed and approved this retrospective study, which was performed in accordance with the principles of the Declaration of Helsinki. Inclusion criteria were patients who had combined cataract extraction and glaucoma drainage device surgery [Baerveldt, Abbott Medical, Abbott Park (IL) or Ahmed valve, New World Medical, Rancho Cucamonga (CA)] between June 2009 and August 2017. All surgeries were performed at the University of Illinois at Chicago (UIC) by two different surgeons. Both surgeons used the same surgical technique described below. Exclusion criteria included pre- and/or postoperative VA of $\leq 20$ / 200 causing inadequate refraction testing and patients who did not undergo manifest refraction because they were lost to follow-up in the postoperative period. 


\section{Data Collection}

Data were obtained through a retrospective chart review and entered into a standard computerized database for subsequent analysis. The data collected included: patient demographics (age, gender, ethnicity), pretreatment refractive findings [visual acuity (VA), refractive error, keratometry $(K)$ values, $\mathrm{AL}, \mathrm{ACD}]$, preoperative IOP, surgical complications, postoperative IOP, and postoperative visual and refractive outcomes. $K$ values were obtained using IOLMaster partial coherence interferometry biometer (Carl Zeiss Meditec AG, Berlin, Germany). AL values were obtained using the IOLMaster partial coherence interferometry biometer or contact A-scan with the Eye Cubed ultrasound system (Ellex, Minneapolis, MN). The same IOLMaster biometer was used for all measurements performed during the study period (2009 through 2017).

\section{IOL Model Selection}

The surgeon selected the IOL power based on results of the $K$ and AL measurements with the devices discussed above. The surgeons' discretion was used to decide which measurements were most appropriate for each given patient. Hoffer $Q$ biometric formula was used for IOL calculations in short eyes $(\mathrm{AL} \leq 22)$, and SRK/ $T$ biometric formula was used for IOL calculations in normal and longer eyes (AL > 22).

\section{Surgical Techniques}

The Baerveldt (Abbott Laboratories Inc., IL) GDD is a non-valved GDD while the Ahmed (New World Medical Inc., CA) GDD is a valved GDD (Fig. 1). The Ahmed shunt tends to work immediately whereas the Baerveldt may take 4-6 weeks to see full effect. Implantations were performed with the implant placed in the superotemporal quadrant, and all implants were placed beneath the respective rectus muscles. All Baerveldt GDDs were ligated with 7-0 Vicryl suture [11]. Cataract surgery was performed by a temporal clear cornea approach, with standard phacoemulsification techniques [11].

\section{Outcome Measures}

The main outcome measure evaluated was whether or not spherical equivalent (SE) between $-1.00 \mathrm{D}$ and $+1.00 \mathrm{D}$ from target refraction was achieved at 3-6 months
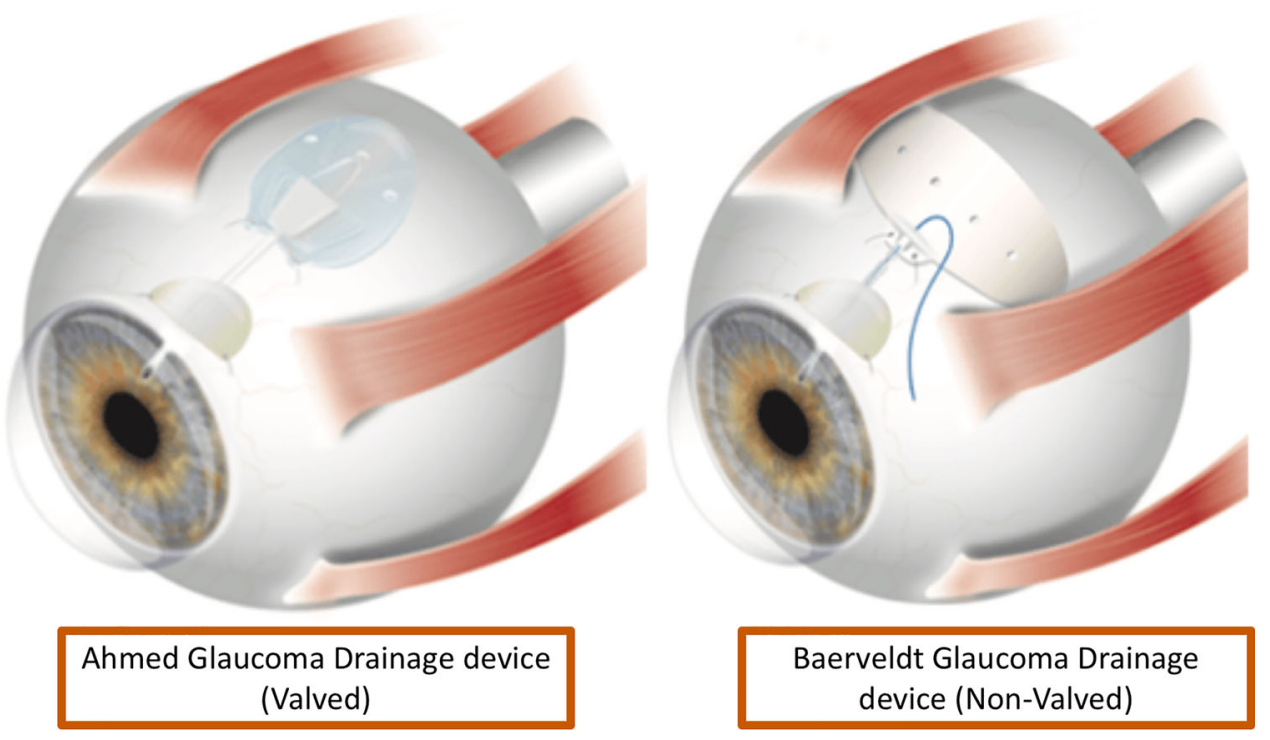

Fig. 1 The Baerveldt GDD is a non-valved GDD while the Ahmed GDD is a valved GDD. Image source: https://www. willseye.org/glaucoma-tube-shunts/ 
postoperatively [11]. Similar to the timeframe reported in previous studies, uncorrected VA was noted to stabilize 3-6 months postoperatively with resolution of early postoperative complications including corneal edema, IOP fluctuations, and hyphema [11]. Manifest refraction was performed for all patients 3-6 months postoperatively. Refractive predictions were based on the preoperative keratometric values and used as a measure of successful refractive outcome. For secondary outcomes, we further assessed demographic and clinical characteristics such as age, change in IOP, preoperative ACD, and preoperative VA, which may have affected refractive outcome and the amount of cylinder induced by combined surgery.

Applied cylinder was calculated by a modified approach; preoperative keratometry was performed with automated devices as previously discussed. Manual refraction was used to estimate keratometry values postoperatively. Pre- and postoperative keratometry values were compared to determine induced astigmatism $[11,12]$.

\section{Statistical Analysis}

All statistical analyses were performed using Stata 15 (StataCorp, College Station, TX) statistical package. Continuous variables were summarized with the use of mean and SD, categorical variables with the use of percentages. Generalized estimation equation (GEE) models, which could account for correlation between two eyes within patients, were used to determine which factors predicted whether a SE of $-1.00 \mathrm{D}$ to $+1.00 \mathrm{D}$ was achieved and to evaluate factors that predicted change in cylinder.

\section{RESULTS}

\section{Study Population}

A total of 63 eyes underwent combined cataract extraction and glaucoma drainage device surgery during the time period specified at this institution. Twenty-one eyes were excluded from the study. Eleven of 21 eyes had pre- and/ or postoperative VA of $\leq 20 / 200$ so these patients could not have refraction adequately tested. Vision loss postoperatively in 11 excluded eyes was attributed to poor preoperative VA $(n=6)$, macular edema and hypotony $(n=3)$, aqueous misdirection $(n=1)$, and progression of glaucoma $(n=1)$, respectively. Ten patients had missing data or were lost to follow-up and did not undergo manifest refraction after surgery. Forty-two eyes of 38 patients were subsequently included in the study for analysis of refractive outcomes. Patient demographics and preoperative and operative data for the excluded and included eyes are summarized in Table 1A, B. No statistically significant difference was found when excluded eyes were compared with the included eyes in all characteristics except VA $(p=0.003)$.

In the final analysis, 42 eyes underwent cataract surgery with Ahmed valve in $17 \%$ of eyes $(n=7)$ and Baerveldt implant in $83 \%$ of eyes $(n=35)$. Mean preoperative $\mathrm{AL}$ was $23.89 \pm 1.26 \mathrm{~mm}$, and preoperative anterior chamber depth (ACD) was $2.96 \pm 0.50 \mathrm{~mm}$.

\section{Refractive Outcomes}

Table 2 summarizes the refractive outcomes, and induced corneal astigmatism at 3 to 6 months postoperatively. A refractive outcome of SE between $-1.00 \mathrm{D}$ and $+1.00 \mathrm{D}$ of the target refraction was achieved in 30 of 42 eyes $(71.43 \%)$ at 3-6 months after surgery.

Mean preoperative AL of eyes with postoperative $S E$ outside $\pm 1 \mathrm{D}$ from target $(\mathrm{SD}=0.98, p=0.003)$ was noted to be $25.37 \pm 0.98 \mathrm{~mm}$ (longer mean $\mathrm{AL}$ ) and that of eyes with SE between $+1.00 \mathrm{D}$ and $-1.00 \mathrm{D}$ $(\mathrm{SD}=0.89, p=0.000)$ was found to be $23.34 \pm 0.89 \mathrm{~mm}$ (average mean $\mathrm{AL}$ ). A regression analysis indicated that longer preoperative $\mathrm{AL}$ noted in 12 eyes was associated with a postoperative hyeropic shift in refractive outcome [0.28 (0.11), $p=0.022]$ compared to 30 eyes with average preoperative $\mathrm{AL}$, which demonstrated a myopic shift in refractive outcome $[-0.15(0.07), p=0.037]$ to refractive 
Table 1 Patient preoperative and operative clinical information for (A) included eyes and (B) excluded eyes

(A) Number of eyes (patients)

$42(38)$

Preoperative BCVA (best corrected visual acuity)

Number (\%)

20/20-20/40

$10(24 \%)$

$20 / 50-20 / 200$

$32(76 \%)$

Glaucoma type (open angle)

$42(100 \%)$

Selected lens type

Monofocal 1-piece SN60

$42(100 \%)$

Location of lens

Capsular bag

Glaucoma surgery drainage device type

Ahmed valve shunt

$7(17 \%)$

Baerveldt glaucoma implant

$35(83 \%)$

Preoperative axial length (mean \pm SD)

$23.89 \pm 1.26$

Preoperative anterior chamber depth (mean \pm SD)

$2.96 \pm 0.50$

B) Number of eyes

21

Preoperative BCVA (best corrected visual acuity)

Number (\%)

20/20-20/40

$2(10 \%)$

$20 / 50-20 / 200$

$9(43 \%)$

Worse than $20 / 200$

$10(47 \%)$

Glaucoma type (open angle)

$21(100 \%)$

Selected lens type

Monofocal 1-piece SN60

$21(100 \%)$

Location of lens

Capsular bag

$21(100 \%)$

Glaucoma surgery drainage device type

Ahmed valve shunt

Baerveldt glaucoma implant

Preoperative axial length (mean \pm SD)

$24.38 \pm 1.53$

$n=11$ (missing data $n=10)$

Preoperative anterior chamber depth (mean \pm SD)

$2.87 \pm 0.39$

$n=11$ (missing data $n=10)$ 
Table 2 Refractive outcomes at 3-6 months postoperatively

Total number of eyes

42

Difference in spherical equivalent between target and final refractive outcome after surgery

Outside $\pm 1.00 \mathrm{D}$

Between $+1.00 \mathrm{D}$ and $-1.00 \mathrm{D}$ $30(71 \%)$

Axial length of eyes with postoperative spherical equivalent

Outside $\pm 1.00 \mathrm{D}($ mean $\pm \mathrm{SD})$

$25.37 \pm 0.98$

Between $+1.00 \mathrm{D}$ and $-1.00 \mathrm{D}$

$23.34 \pm 0.89$

$($ mean $\pm \mathrm{SD})$

Number of eyes with postoperative

$12(29 \%)$

induced cylinder

$0.52 \pm 0.49$

Amount of induced cylinder

$($ mean $\pm \mathrm{SD})$

outcome in 30 eyes with difference between $-1.00 \mathrm{D}$ and $+1.00 \mathrm{D}$ of the target refraction (Fig. 2).

Pre- and postoperative keratometry values were compared for all 42 eyes, while most eyes had no induced astigmatism; 12 eyes (29\%) were noted to have a mean $0.52 \mathrm{D}(\mathrm{SD}=0.49$; range $0.02-1.49)$ of corneal astigmatism induced by combined surgery. Age $(p=0.905)$, ethnicity $(p=0.314)$, gender $(p=0.825)$, preoperative ACD $(p=0.302)$, preoperative intraocular pressure (IOP) $(p=0.374)$, and postoperative IOP $(p=0.177)$ did not significantly affect refractive outcomes in the sample.

\section{DISCUSSION}

Patients with advanced glaucoma and visually significant cataracts often require a combined surgical approach with cataract extraction and GDD surgery. This is the largest study to report refractive outcomes in patients undergoing this combined surgical approach. We evaluated 42 eyes of 38 patients who underwent combined cataract extraction and glaucoma drainage device surgery. Favorable refractive outcomes (SE between $+1.00 \mathrm{D}$ and $-1.00 \mathrm{D}$ from target) were achieved in $71 \%$ of patients despite the potential alteration of preoperative measurements and introduction of error into lens selection when using a combined approach.

Previous studies by Law et al. provided data regarding refractive outcomes of a combined cataract extraction and trabeculectomy. They found the difference in mean refractive error of the combined operation group (0.22D) and cataract operation only group (0.29D) was not statistically significant $(P=0.80)$, despite changes in AL and corneal curvature postoperatively [10]. Recognizing the need to explore refractive outcomes in patients who have undergone combined cataract extraction and GDD surgery, Tzu et al. studied these outcomes in 21 eyes that underwent combined cataract extraction with GDD surgery. This subset comprised $49 \%$ of their study population. The remaining $51 \%$ of eyes $(n=22)$ underwent combined cataract extraction and trabeculectomy surgery. Favorable refractive outcomes (SE between $+1.00 \mathrm{D}$ and $-0.50 \mathrm{D}$ from target) were reported in most patients, regardless of type of glaucoma surgery. Seventy-seven percent of combined trabeculectomy (17 of 22 ) and $71 \%$ of combined GDD (15 of 21) were within acceptable range. The authors acknowledged that a larger sample size would allow for a better understanding of the factors influencing these refractive results [11]. A larger sample size in our study confirms that most eyes undergoing combined cataract extraction and GDD surgery achieve favorable refractive outcomes postoperatively (within \pm 1D of target SE).

Our study evaluated characteristics and factors associated with refractive surprise. All patients underwent manifest refractions at the 3-6 month postoperative timeframe because previous studies have reported that uncorrected VA stabilizes 3-6 months postoperatively with resolution of early postoperative complications including corneal edema, IOP fluctuations, and hyphema [11]. In the present study, 12 patients who fell outside of the target range were in the "outside $\pm 1.0 \mathrm{D}$ " category at 3-6 months postoperatively, which implied that their 

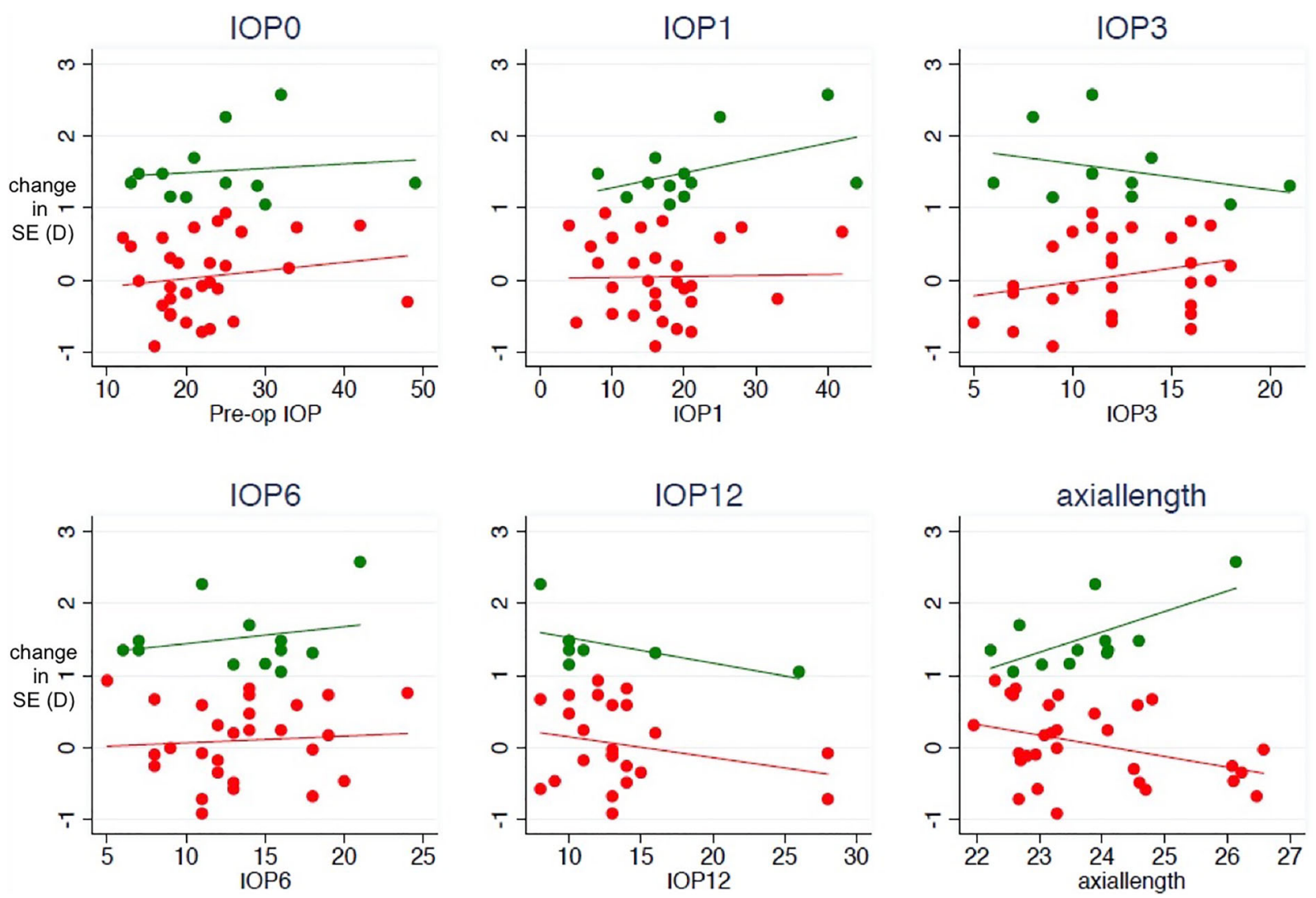

Fig. 2 Regression analysis showing relation between difference in refractive outcome [spherical equivalent (SE) in diopters (D)] and preoperative intraocular pressure (IOP0); postoperative IOP at 1 month (IOP1), 3 months (IOP3), 6 months (IOP6), and 12 months (IOP12) after

postoperative refractive outcome was more myopic than the predicted refractive goal. Preand postoperative IOP did not significantly affect refractive outcomes in the sample (Fig. 2). Preoperative AL was positively related to refractive outcome in eyes with difference in spherical equivalent outside $\pm 1.00 \mathrm{D}$ compared to the target refraction, but was negatively related to refractive outcome in eyes with difference in spherical equivalent between $-1.00 \mathrm{D}$ and + $1.00 \mathrm{D}$ compared to target refraction, and this was statistically significant (Fig. 2). Postoperative hyperopic refractive surprise was associated with longer $\mathrm{AL}$; in our study the mean preoperative AL of eyes with postoperative SE outside $\pm 1.00 \mathrm{D}$ was $25.37 \pm 0.98 \mathrm{~mm}$ compared to the preoperative $\mathrm{AL}$ of eyes with $\mathrm{SE}$ between $+1.00 \mathrm{D}$ and $-1.00 \mathrm{D}$, which was surgery; and preoperative axial length. Red data points $=$ 30 eyes with final refractive outcome at goal $( \pm 1.00 \mathrm{D}$ of the target refraction). Green data points $=12$ eyes with final refractive outcome outside goal $(> \pm 1.00 \mathrm{D}$ of the target refraction)

$23.34 \pm 0.89 \mathrm{~mm}$. The tendency for a patient to have postoperative hyperopic surprise when axial length falls outside of the acceptable range is consistent with the fact that $\mathrm{AL}$ decreases after GDD surgery [3]. Decreases in AL by $0.1-0.9 \mathrm{~mm}$ have been reported after glaucoma surgery $[2,8,13-15]$. The AL is thought to be affected by external flow of aqueous humor through the glaucoma drainage device, shallowing of the anterior chamber, and possibly a thickened choroid accompanying a low IOP after glaucoma surgery. Cashwell et al. showed that there was an average decrease of $0.46 \mathrm{~mm}$ in $\mathrm{AL}$ after trabeculectomy in phakic eyes, which then increased an average of $0.275 \mathrm{~mm}$ after cataract surgery [3]. Francis et al. reported a statistically significant reduction in $\mathrm{AL}$, which was similar after trabeculectomy and GDD 
surgery at all time points, and at 3 months or later the AL reduction was related to postoperative IOP and the amount of IOP reduction [16]. Given the retrospective nature of our study where postoperative AL measurements were not obtained after combined cataract extraction and GDD surgery, the postoperative changes in $\mathrm{AL}$ in our patients could not be factored into our evaluation.

While AL is one biometric feature of the eye affected by filtering glaucoma surgery, another biometric property that is affected is the ACD. Ning et al. studied the postoperative changes in ACD after cataract surgery and found that ACD deepened after 2 weeks and the change was larger in eyes with preoperative shallow anterior chamber $(<2.5 \mathrm{~mm})$ and short $\mathrm{AL}(<23 \mathrm{~mm})$. This deepened ACD was associated with refractive error after cataract surgery [17]. In glaucoma surgery, the ACD fluctuates postoperatively. Ishida et al. reported a transient decrease in ACD 4 weeks after glaucoma filtering surgery with the EX-PRESS device [18]. Diagourtas et al. noted that ACD after trabeculectomy fluctuates with changes in intraocular pressure [19]. In contrast, Miraftabi et al. studied the changes in ocular biometric properties after Ahmed glaucoma valve surgery and noted that AL decreased 3 months after surgery but ACD was not affected [20]. In our study, mean preoperative ACD was measured with mean $2.96 \pm 0.50 \mathrm{~mm}$ but postoperative ACD was not measured. Postoperative ACD changes have not been previously studied in combined cataract and glaucoma surgerylikewise, due to the retrospective nature of our study, we were unable to include this assessment as part of our understanding of refractive outcomes after surgery.

Another component of the postoperative refractive outcome, which needs further investigation, is surgically induced astigmatism. Trabeculectomy, particularly fornix-based trabeculectomy surgery, is associated with withthe-rule astigmatic change in the immediate postoperative period, followed by a gradual against-the-rule shift. Phacoemulsification alone with a temporal clear cornea approach has been reported to induce a mean postoperative with-the-rule astigmatism of $0.768 \mathrm{D}$ after 3 months but there is no clear evidence regarding astigmatism induced by glaucoma tube shunt surgery $[21,22]$. In our study, most eyes did not experience induced corneal astigmatism after combined cataract and GDD surgery; 29\% eyes $(n=12)$ were noted to have a mean $0.52 \mathrm{D}$ $(\mathrm{SD}=0.49)$ of corneal with-the-rule astigmatism induced by combined surgery, which was likely a result of the temporal clear corneal main wound created for the phacoemulsification component of the combined surgery. While unable to directly compare these results to prior studies given the difference in surgical techniques, Law et al. reported 0.44D mean induced astigmatism after combined cataract extraction and fornix-based trabeculectomy surgery while Tzu et al. reported $1.31 \pm 0.86 \mathrm{D}$ induced astigmatism in their study population, which comprised eyes that had undergone cataract extraction with GDD or trabeculectomy $[10,11]$. The pre- and postoperative astigmatism reported in our study was based on the manifest refraction performed 3-6 months after surgery. As a result, the preoperative astigmatism measurement included corneal and lenticular astigmatism while the postoperative measurements were reflective of corneal astigmatism alone. Given the retrospective nature of our study, postoperative corneal topography was not available for review but future study designs should plan to obtain pre- and postoperative corneal topography to better understand induced corneal astigmatism in patients undergoing combined cataract and GDD surgery.

This is the largest study to date to evaluate factors affecting postoperative refractive outcomes in eyes that have undergone combined cataract removal and GDD surgery. This study analyzed the relationship between preoperative $\mathrm{AL}$ and postoperative refractive outcomes and found that longer preoperative ALs were noted in patients with refractive change $>1 \mathrm{D}$ from target refraction. Eyes with longer mean $\mathrm{AL}$ demonstrated a hyperopic shift in refractive outcome, while eyes with average preoperative ALs were associated with a myopic shift in refractive outcome. The findings highlighted in this study can help guide patient education, IOL 
selection, and the consent process in the preoperative period.

Limitations of the study include the retrospective nature of the study resulting in missing data, which results in reducing size and power of the study, and lack of an appropriate control group. The retrospective design also limited our ability to measure postoperative AL and postoperative $\mathrm{ACD}$ at the time of postoperative refraction to better understand how they influence refractive outcomes after combined cataract extraction and glaucoma surgeries. Obtaining formal corneal topography during the postoperative period would also allow better understanding of induced astigmatism in patients undergoing combined cataract and GDD surgery. These additional measures would further our understanding of the observed postoperative hyperopic and myopic shifts and induced astigmatism.

\section{CONCLUSION}

In summary, patients with advanced glaucoma and visually significant cataracts often need a combined surgical approach with cataract extraction and GDD surgery, and our study found that favorable refractive outcomes can be achieved in most patients undergoing such combined surgeries.

\section{ACKNOWLEDGMENTS}

Funding. The study was supported by NEI K23EY022949 (T.S.V.), University of Illinois Core grant EY49570, Komarek-Hyde-McQueen Foundation, Northbrook, IL, and Illinois Society for the Prevention of Blindness, New York, NY.

Prior Presentation. Presented at the 2019 ARVO Annual Meeting, May 1, 2019, Vancouver Canada.

Authorship. All named authors meet the International Committee of Medical Journal Editors (ICMJE) criteria for authorship for this article, take responsibility for the integrity of the work as a whole, and have given their approval for this version to be published.

Author Contributions. All authors contributed to the study conception and design. Material preparation, data collection and analysis were performed by Shizuka Tomatzu and Dingcai Cao. The first draft of the manuscript was written by Rajvi Mehta and all authors commented on previous versions of the manuscript. All authors read and approved the final manuscript.

Disclosures. Rajvi Mehta, Shizuka Tomatzu, Dingcai Cao, Alexander Pleet, Alexander Mokhur, Ahmad A. Aref, Thasarat Sutabutr Vajaranant declare no conflict of interest.

Compliance with Ethics Guidelines. The University of Illinois (UIC) Institutional Review Board reviewed and approved this retrospective study, which was performed in accordance with the principles of the Declaration of Helsinki.

Data Availability. The datasets generated during and/or analyzed during the current study are available from the corresponding author on reasonable request.

Open Access. This article is licensed under a Creative Commons Attribution-NonCommercial 4.0 International License, which permits any non-commercial use, sharing, adaptation, distribution and reproduction in any medium or format, as long as you give appropriate credit to the original author(s) and the source, provide a link to the Creative Commons licence, and indicate if changes were made. The images or other third party material in this article are included in the article's Creative Commons licence, unless indicated otherwise in a credit line to the material. If material is not included in the article's Creative Commons licence and your intended use is not permitted by statutory regulation or exceeds the permitted use, you will need to obtain permission directly from the copyright holder. To view a copy of this licence, visit http://creativecommons.org/licenses/by$\mathrm{nc} / 4.0 /$. 


\section{REFERENCES}

1. Tan HY, Wu SC. Refractive error with optimum intraocular lens power calculation after glaucoma filtering surgery. J Cataract Refract Surg. 2004;30: 2595-7.

2. Hugkulstone CE. Changes in keratometry following trabeculectomy. Br J Ophthalmol. 1991;75:217-8.

3. Cashwell LF, Martin CA. Axial length decrease accompanying successful glaucoma filtration surgery. Ophthalmology. 1999;106:2307-11.

4. Kook MS, Kim HB, Lee SU. Short-term effect of mitomycin-C augmented trabeculectomy on axial length and corneal astigmatism. J Cataract Refract Surg. 2001;27:518-23.

5. Uretmen $\mathrm{O}$, Ates $\mathrm{H}$, Andac $\mathrm{K}$, et al. Axial length changes accompanying successful non-penetrating glaucoma filtration surgery. Ophthalmologica. 2003;217:199-203.

6. Claridge KG, Karmel GV, Bates AK. The effect of trabeculectomy on refraction, keratometry and corneal topography. Eye. 1995;9:292-8.

7. Kao SF, Lichter PR, Musch DC. Anterior chamber depth following filtration surgery. Ophthalmic Surg. 1989;20:332-6.

8. Rosen WJ, Mannis MJ, Brandt JD. The effect of trabeculectomy on corneal topography. Ophthalmic Surg. 1992;23:395-8.

9. Francis BA, Wang $\mathrm{M}$, Lei $\mathrm{H}$, et al. Changes in axial length following trabeculectomy and glaucoma drainage device surgery. Br J Ophthalmol. 2005;89: 17-20.

10. Law SK, Manusry AM, Vasudev D, et al. Effects of combined cataract surgery and trabeculectomy with mitomycin $\mathrm{C}$ on ocular dimensions. Br J Ophthalmol. 2005;89:1021-5.

11. Tzu JH, Shah CT, Galor A, Junk AK, Sastry A, Wellik SR. Refractive outcomes of combined cataract and glaucoma surgery. J Glaucoma. 2015;24(2):161-4.

12. Cravy TV. Calculation of the change in corneal astigmatism following cataract extraction. Ophthalmic Surg. 1979;10:38-49.
13. Nemeth J, Horoczi Z. Changes in the ocular dimensions after trabeculectomy. Int Ophthalmol. 1992;16:355-7.

14. Claridge KG, Galbraith JK, Karmel V, et al. The effect of trabeculectomy on refraction, keratometry and corneal topography. Eye. 1995;9(Pt 3):292-8.

15. Vernon SA, Zambarakji HJ, Potgieter F, et al. Topographic and keratometric astigmatism up to 1 year following small flap trabeculectomy (microtrabeculectomy). Br J Ophthalmol. 1999;83:779-82.

16. Francis BA, Wang $\mathrm{M}$, Lei $\mathrm{H}$, et al. Changes in axial length following trabeculectomy and glaucoma drainage device surgery. $\mathrm{Br} \mathrm{J}$ Ophthalmol. 2005;89(1):17-20.

17. Ning X, Yang Y, Yan H, Zhang J. Anterior chamber depth-a predictor of refractive outcomes after agerelated cataract surgery. BMC Ophthalmol. 2019;19(1):134.

18. Ishida K, Moroto N, Murata K, Yamamoto T. Effect of glaucoma implant surgery on intraocular pressure reduction, flare count, anterior chamber depth, and corneal endothelium in primary open-angle glaucoma. Jpn J Ophthalmol. 2017;61(4):334-46.

19. Diagourtas A, Papaconstantinou D, Vergados A, Andreanos K, Koutsandrea C. Objective documentation of anterior chamber depth following trabeculectomy and its correlation with intraocular pressure and bleb functionality. Medicine. 2018;97(34):11824.

20. Miraftabi A, Lotfi M, Nilforushan N, Abdolalizadeh P, Jafari S. Ocular biometric changes after Ahmed glaucoma valve implantation. Eur J Ophthalmol. 2019;21:112.

21. Chan HHL, Kong YXG. Glaucoma surgery and induced astigmatism: a systematic review. Eye Vis (Lond). 2017;4:27.

22. Nikose AS, Saha D, Laddha PM, Patil M. Surgically induced astigmatism after phacoemulsification by temporal clear corneal and superior clear corneal approach: a comparison. Clin Ophthalmol. 2018;12:65-70. 